Monográfico: ENDOUROLOGÍA Y LÁSER

Arch. Esp. Urol., 61, 9 (1.103-1.110), 2008

\title{
LITIASIS EN CÁLIZ INFERIOR: UTILIDAD DEL LÁSER
}

Almudena Coloma del Peso, Inmaculada Fernández González, Pablo Garrido Abad, Milagros Jimenez Galvezl, Gloria Bocardo Fajardo, Luis Miguel Herranz Fernándezl, Miguel Mora Durbán, Jerónimo Muñoz-Delgado Salmerón, Alvaro Serrano Pascual², Lorenzo Herrero Torres e Ignacio Pereira Sanz.

Servicio de Urología. Hospital Universitario de la Princesa. Madrid

'Servicio de Urología. Hospital del Henares. Madrid

${ }^{2}$ Servicio de Urología. Hospital Universitario de Guadalajara. España.

Resumen.- Todavía permanece en controversia cual es la mejor modalidad de tratamiento para las litiasis localizadas en el cáliz inferior. El rango de aclaramiento litásico de los cálculos situados en el cáliz inferior va a depender de diferentes factores como el tamaño y la composición del cálculo, el tipo de litotriptor utilizado, el tipo de transporte urinario y la anatomía del cáliz inferior. El papel de la Ureteroscopia (URS) flexible en el tratamiento de la patología intrarrenal ha experimentado una dramática evolución, impulsada por las mejoras en el diseño de los ureteroscopios flexibles, en su grado de deflexión y mejora de la calidad de imagen, en la gran diversificación de la intrumentación accesoria de pequeño calibre y en el uso del láser de Holmium (Ho: YAG) para la litotricia. Su desarrollo permite ofrecerla como modalidad terapeútica en los fracasos de la Litotricia Extracorpórea (LEC) en litiasis menores de $1 \mathrm{~cm}$ y como primera línea de tratamiento en las litiasis menores de $1 \mathrm{~cm}$ en casos de cálculos de cistina y en aquellos con niveles de atenuación mayores a $1000 \mathrm{HU}$; asi como en pacientes obesos o con problemas de coagulación.

Palabras clave: Litiasis cáliz inferior. Litotricia extracorpórea. Láser.

Summary.- There is controversy yet about which is the best treatment modality for lithiasis of the lower calyx. The range of lithiasis clearance of the stones localized in the lower calyx will depend on various factors such as size, composition of the stone, type of lithotripter employed, type of urinary transport, and anatomy of the lower calyx. The role of flexible ureteroscopy in the treatment of intrarenal pathology has experimented a dramatic evolution, impulsed by the improvements in design of flexible ureterscopes, their degree of deflection, and better quality of image, in the great diversification of small calibre accessory instruments, and the use of the holmium laser for lithotripsy. Its development makes possible to offer it as a therapeutic option for the failures of extracorporeal lithotripsy in stones smaller than $1 \mathrm{~cm}$ and as first-line treatment for stones smaller than $1 \mathrm{~cm}$ in size if they are cystine stones or they have an attenuation level over $1000 \mathrm{HU}$; also in obese patients or those with coagulation problems.

Keywords: Lower calyx lithiasis. Extracorporeal lithotripsy. Laser. 


\section{INTRODUCCIÓN}

Todavía permanece en controversia cual es la mejor modalidad de tratamiento para las litiasis localizadas en el cáliz inferior. El rango de éxito de la Litotricia Extracorpórea (LEC) es del $63 \%$ al $74 \%$ en los cálculos menores de $1 \mathrm{~cm}$ y del 23 al $56 \%$ en los cálculos con un tamaño de $1-2 \mathrm{~cm}$ (1-5). El bajo rango de aclaramiento litásico de los cálculos situados en el cáliz inferior va a depender de diferentes factores como son el tamaño y la composición del cálculo, el tipo de litotriptor utilizado, el tipo de transporte urinario y la anatomía del cáliz inferior (6-9).

\section{FACTORES QUE AFECTAN AL RANGO DE "STONE FREE"}

\section{COMPOSICIÓN}

Recientemente, los niveles de atenuación de la Tomografía Axial Computerizada (TAC) han sido utilizados para diferenciar la composición de la litiasis. Estudios "in vitro" muestran que los niveles de atenuación para el ácido úrico y la estruvita es menor de 1000 unidades Hounsdfield $(\mathrm{HU})$, mientras que el oxalato cálcico y la hidroxiapatita habitualmente exceden de $1000 \mathrm{HU}(10,11)$. Joseph y cols. informaron de un rango de aclaramiento litiasico con la LEC del $54.5 \%$ para niveles de atenuación mayores a $1000 \mathrm{HU}$ frente al $85,7 \%$ para niveles de atenuación entre 500 y $1000 \mathrm{HU}$ y al $100 \%$ para niveles de atenuación menores de $500 \mathrm{HU}$ (12). Gupta y cols., utilizan $750 \mathrm{HU}$ como punto de corte y el rango de aclaramiento litiásico es del $65 \%$ para los cálculos con niveles de atenuación mayor de $750 \mathrm{HU}$ frente al $90 \%$ para aquellos con niveles de atenuación menor de $750 \mathrm{HU}$ (13).

\section{TIPO DE LITOTRIPTOR UTILIZADO}

El tipo de litotriptor utilizado influye en el resultado de la LEC ya que se ha demostrado que los litotriptores de primera generación son los más efectivos. Sampaio y cols.

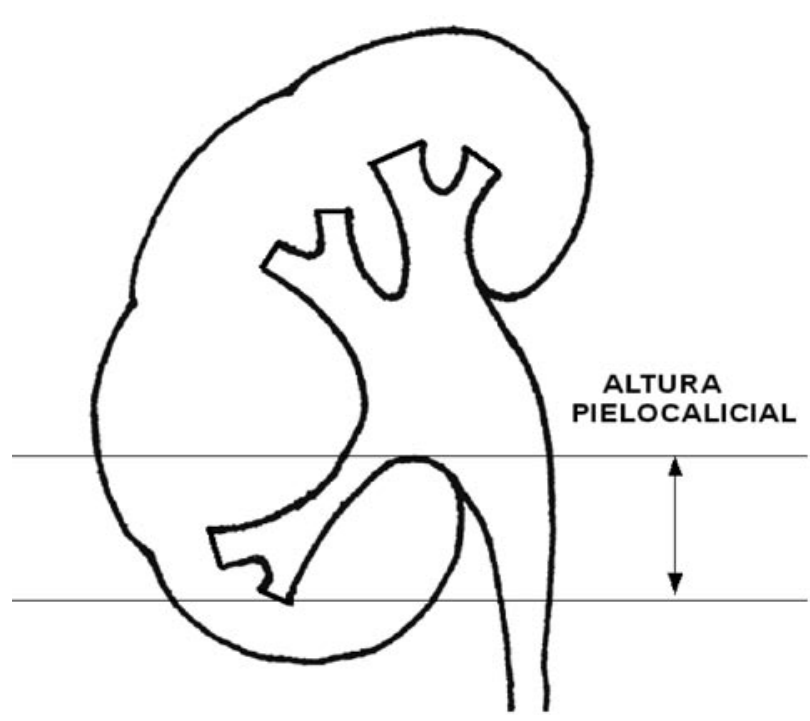

informan un rango de "stone-free" del 59,5\% con el litotriptor Lithostar Plus (Siemens Medical Systems, Iselin, New Jersey) (14). Elbahnasy y cols. refieren un rango de "stone free" del 64\% con el HM-3 (Dornier Medical Systems) $168 \%$ para litiasis menores de $1 \mathrm{~cm}$ y $59 \%$ para las litiasis entre 1 y $2 \mathrm{~cm}$ ) (15). May y Chandhoke informan un rango de "stone-free" del 75\% (76\% para las litiasis del cáliz inferior de $1 \mathrm{~cm}$ ó menores y del $74 \%$ para litiasis del cáliz inferior de 11 a $20 \mathrm{~mm}$ )con el HM-3 (16).

Otro factor importante a tener en cuenta es la distancia de la piel al cálculo medida con la TAC sin contraste, poniendo el punto de corte en $10 \mathrm{~cm}$ enfatizando la importancia de la existencia de una máxima distancia focal de los litotriptores en el rango de "stone free" (17).

\section{TIPO DE TRANSPORTE URINARIO}

El concepto del tipo transporte urinario fue introdcido por Schulz y cols. que describen las características dinámicas del mismo en el sistema pielocalicial (18).

Existen diferentes tipos de transporte urinario. Los tipos 1 y 2 son fisiológicos. En el tipo 1 las ondas peristálticas se originan en el cáliz del grupo calicial superior y son transmitidas a la pelvis renal; el reflujo de orina en el grupo calicial inferior inicia una onda que estimula el paso de la orina a traves de la unión pieloureteral y un flujo retrógrado al grupo calicial superior. En el tipo 2, las ondas peristálticas se inician simultanemente en todo el sistema colector originando un flujo anterógrado al uréter y cierto flujo retrógrado calicial. El tipo 3 y 4 son patológicos. En el tipo 3 las ondas peristálticas de baja intensidad se desplazan a una pelvis con una motilidad disminuida y excreción retardada; en el tipo 4 no hay motilidad ni en la pelvis ni en los cálices.

Madbouly y cols. sugirieron una relación entre el tipo de transporte urinario y el aclaramiento litiásico (19). La asociación entre el aclaramiento litiásico y el tipo de transporte urinario podría explicarse por el origen de las ondas peristálticas generadas por los esfínteres de los cálices menores y mayores. Un mayor número de cálices en un

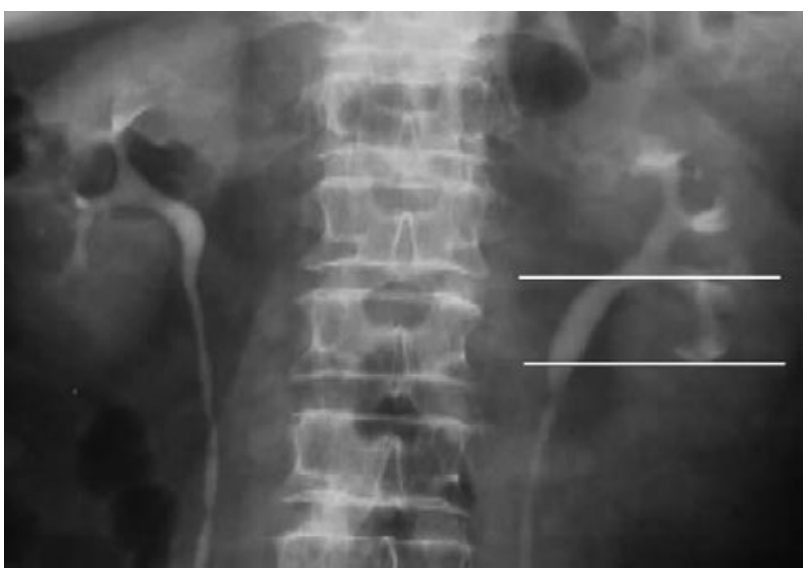

FIGURA IB. Altura pielocalicial. 
sistema colector tipo dendrítico no dilatado puede generar ondas peristálticas más fuertes permitiendo una propulsión más eficiente de orina resultando en un mejor aclaramiento litiásico.

\section{ANATOMÍA DEL CÁLIZ INFERIOR}

\section{- Altura pielocalicial}

Es la distancia entre una línea horizontal que pasa por el punto más inferior del cáliz que contiene la litiasis y otra línea que pasa por el punto más alto del borde inferior de la pelvis renal (CPH) (Figuras 1A y 1B). Para Poulasky y cols. es uno de los más fuertes predictores del aclaramiento litiásico. Una altura pielocalicial menor de $15 \mathrm{~mm}$ se asocia con un mayor rango de aclaramiento litiásico (20).

\section{- Longitud infundibular}

Es la distancia entre la parte más distal del cáliz que contiene al cálculo y el punto medio del borde inferior de la pelvis renal. Cuando la longitud infundibular es mayor de $3 \mathrm{~cm}$ el rango de "stone free" disminuye aproximadamente un $30 \%$ en los pacientes tratados con LEC (21). (Figuras $2 \mathrm{~A}$ y $2 \mathrm{~B}$ )

\section{- Anchura infundibular}

Sampaio y cols. demuestran que el $60 \%$ de los riñones normales anatómicamente disecados tienen un diámetro infundibular mayor de $4 \mathrm{~mm}$ (22). Elbahnasy y cols. informan de que en los pacientes con un diámetro infundibular menor de $5 \mathrm{~mm}$ el rango de "stone free" decrece al $33 \%$ (21) (Figuras 3A y $3 \mathrm{~B}$ ).

\section{- Angulo infundibulopiélico}

Existen diferentes formas de medir este ángulo:

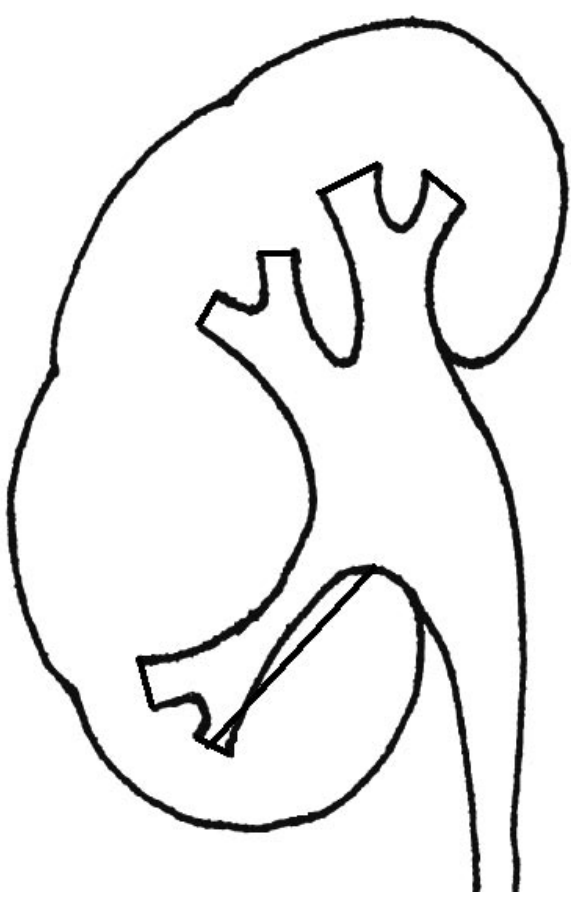

FIGURA 2A. Longitud infundibular.
- Definido por Elbanassy y cols. es el ángulo formado por la intersección del eje ureteropielico y el eje central del infundibulo inferior (21) (Figuras 4A y 4B).

- Utilizando el eje ureteral vertical (8) (Figura 5).

- Utilizando el eje de la pelvis renal (23) (Figura 6).

Se ha observado que un ángulo infundibulo-piélico menor de $90^{\circ}$ afecta desfavorablemente al rango de éxito de la LEC (21-23).

\section{TRATAMIENTO}

\section{- Observación}

Las indicaciones para el tratamiento de la litiasis calicial son el aumento del tamaño del cálculo, la existencia de obstrucción localizada, la asociación con infección y/o ser la causa de un dolor crónico o agudo (24-26). Las indicaciones de tratamiento para las litiasis del grupo calicial inferior son las mismas que para otras localizaciones caliciales. Sin embargo, no existe consenso en el tiempo adecuado para el tratamiento, ni en el tipo de tratamiento para litiasis de pequeño tamaño, no obstructivas y asintomáticas del polo inferior. En un estudio prospectivo randomizado con 2,2 años de seguimiento Keely y cols., comparando litiasis menores de $15 \mathrm{~mm}$ asintomáticas, no encontraron diferencias significativas entre la LEC y la observación en cuanto al rango de "stone free", síntomas, necesidad de tratamientos adicionales, calidad de vida, función renal o necesidad de tratamientos hospitalarios (27).

Hubner y cols., en un estudio retrospectivo, informan que se desarrolla infección en el $68 \%$ de los pacientes con litiasis asintomáticas y que en el $45 \%$ aumenta el tamaño de la litiasis con 7,4 años de seguimiento, concluyendo que el $83 \%$ de los casos podrían precisar tratamiento dentro de los primeros 5 años de diagnóstico (28). Glowazky y cols., en un estudio de cohorte, observan que en el $38 \%$ se desarrolla un evento sintomático con 32 meses de se-

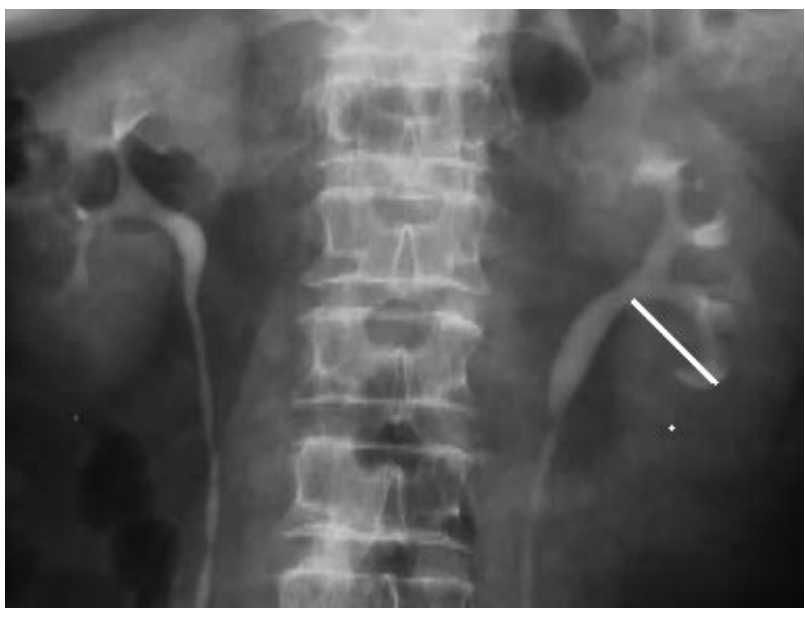

FIGURA 2B. Longitud infundibular. 
guimiento y que la probabilidad acumulada a los 5 años de que ocurra un evento sintomático es del $48,5 \%$ y por ello también recomiendan un tratamiento profiláctico, sin considerar evento sintomático el crecimiento de la litiasis o la asociación a infección (29). En un estudio retrospectivo realizado por Burgher y cols. el $66 \%$ mostraban un aumento del tamaño de la litiasis (30). En un estudio prospectivo de Inci y cols. concluyen que la observación se puede plantear al paciente con litiasis caliciales asintomáticas del grupo callicial inferior pero que en el $33 \%$ van a presentar progresión de la enfermedad y el $11 \%$ va a precisar de otros tatamientos más invasivos a largo plazo (31).

\section{- Litotricia Extracorpórea}

La LEC sigue siendo el tratamiento de elección para la mayoría de los cálculos renales con un tamaño menor de $2 \mathrm{~cm}$. El éxito del tratamiento va a depender de la localización de la litiasis, alcanzando un rango de "stone free" del $56 \%$ al $80 \%$ cuando se sitúan en la pelvis renal frente a otras localizaciones (32). En un estudio multiinstitucional y prospectivo, el rango de "stone free" de la LEC para litiasis mayores de $1 \mathrm{~cm}$ del polo inferior, fue del $21 \%$ frente al $67 \%$ cuando la litiasis era menor de $1 \mathrm{~cm}$ 5. Pearle y cols. no encontraron diferencias en el rango de "stone free" en las litiasis del polo inferior menores de $1 \mathrm{~cm}$ entre la LEC (35\%) y la Ureteroscopia (URS) (50\%).

En este estudio se demostró que los pacientes que se sometieron a ESWL tenían un menor tiempo de convalecencia, precisaban de menor analgesia y probablemente elegirían el mismo procedimiento en comparación con los pacientes que se sometieron a URS (33). Basados en los resultados del meta-análisis de Lingenman y cols., mostrando un rango de "stone free" mayor para la Nefrolitotomía Percutánea (NLPC) que para la LEC para el tratamiento de

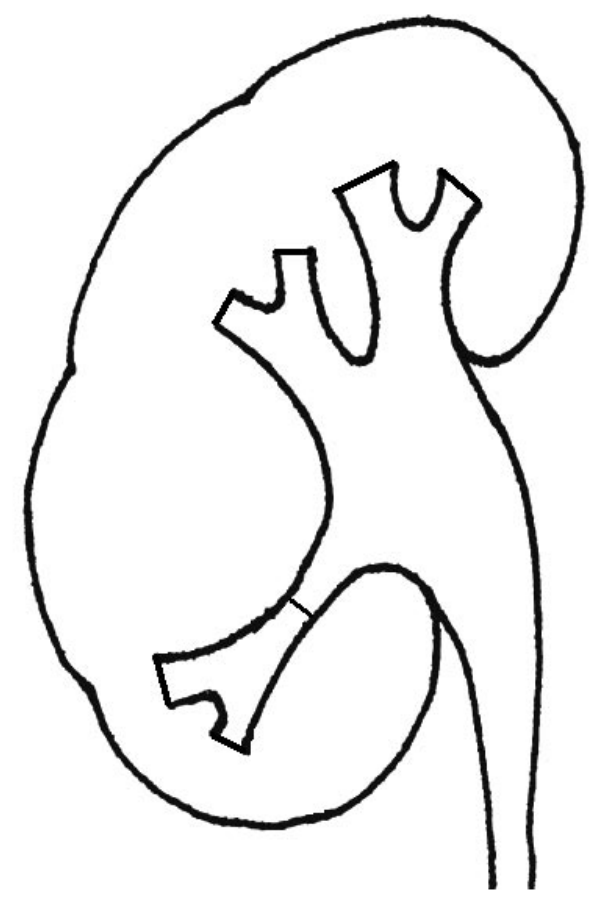

FIGURA 3A. Anchura infundibular. las litiasis del polo inferior de diferente tamaño y validado por estudios prospectivos randomizados, se sugirió que los pacientes con litiasis mayores de $1 \mathrm{~cm}$ deberían ser tratados con NLPC y los de $1 \mathrm{~cm}$ o menos con LEC. El rango de "stone free" fue del $37 \%$ para la LEC frente al $95 \%$ para la NLPC. Cuando se estratifica por tamaños, el rango de "stone free" para litiasis menores de $1 \mathrm{~cm}$ fue del $63 \%$ y $100 \%$ respectivamente; entre $1-2 \mathrm{~cm}$ fue $23 \%$ frente a $93 \%$ y entre 2 y $3 \mathrm{~cm}, 14 \%$ frente al $86 \%$ respectivamente $(4,5)$.

Con estos estudios se concluye que en las litiasis menores de $1 \mathrm{~cm}$ del polo inferior la primera indicación de tratamiento es la LEC y si fracasa la URS.

\section{- Ureteroscopia flexible retrógrada}

El papel de la URS flexible en el tratamiento de la patología intrarrenal ha experimentado una dramática evolución, impulsada por las mejoras en el diseño de los ureteroscopios flexibles, en su grado de deflexión y mejora de la calidad de imagen, en la gran diversificación de la intrumentación accesoria de pequeño calibre y en el uso del láser de Holmium YAG (Ho: YAG) para la litotricia.

Grasso y Ficazzola, informan que el rango general de "stone free" de la URS para el tratamiento de las litiasis del polo inferior menores de $1 \mathrm{~cm}$, entre 1 y $2 \mathrm{~cm}$ y mayores de $2 \mathrm{~cm}$ es del $82 \%, 72 \%$ y $65 \%$, respectivamente; la fragmentación inicial de las litiasis mayores de $2 \mathrm{~cm}$ fue solo del $45 \%$ y el mayor rango de éxito se consiguió realizando el tratamiento en 2 tiempos (34).

Más recientemente, un estudio randomizado comparando los resultados de la ureteroscopia y la LEC en el tratamiento de litiasis menores o iguales a $1 \mathrm{~cm}$, el rango de "stone free" es del $50 \%$ y $35 \%$ respectivamente; los autores sugieren que este bajo porcentaje en ambos grupos se podría justificar por el uso de la TAC que ofrece mayor sensibilidad para detectar los cálculos (33). Se ha sugerido que aquellos pacientes con una anatomía del polo inferior desfavorable para la LEC(ángulo infundibular < $90^{\circ}$, longitud infundibular $>3 \mathrm{~cm}$ o anchura infundibular $<5 \mathrm{~mm}$ ) se podrían tratar mejor con URS $(15,21)$, aunque Grasso

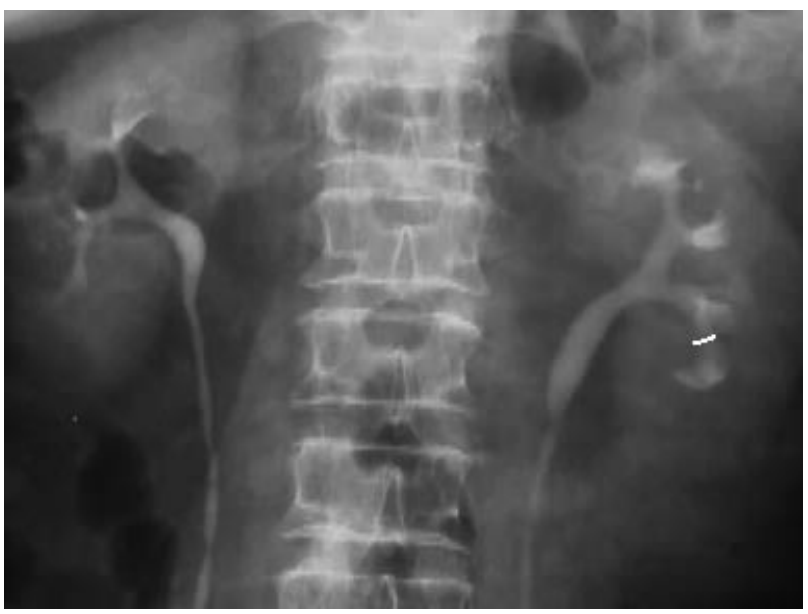

FIGURA 3B. Anchura infundibular. 


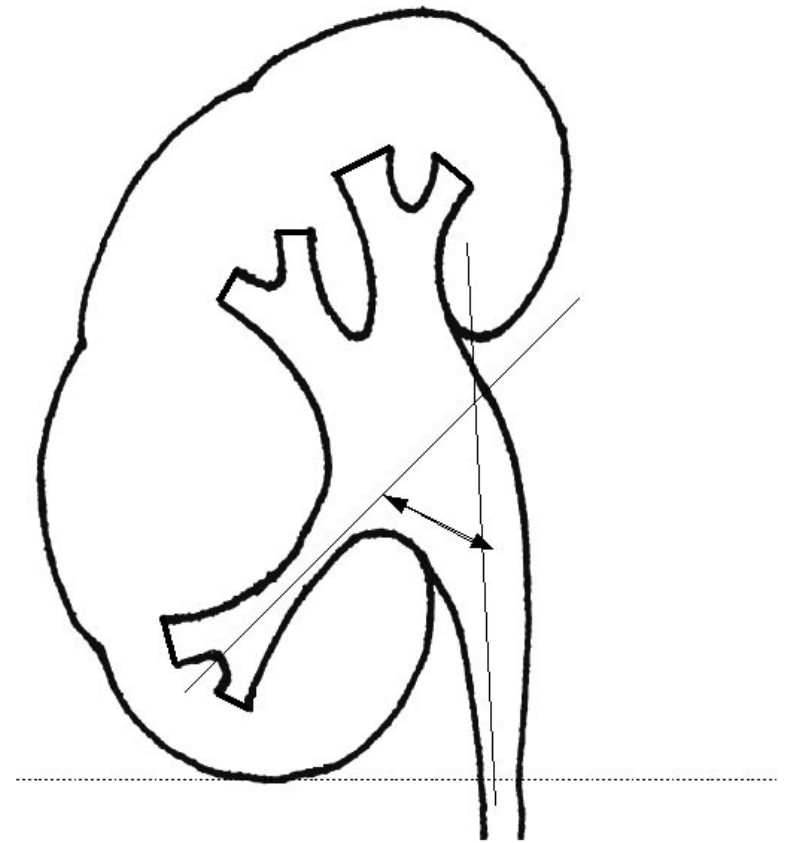

FIGURA 4. Ángulo infundibulo-piélico de Elbanhasy.

y Picazzola creen que los factores anatómicos que pueden afectar los resultados negarivos de la LEC tambien pueden disminuir de forma significativa el rango de éxito de la URS (34).

Para la realización de la ureteroscopia flexible el paciente se coloca en posición de litotomía modificada, aunque se han propuesto otras posiciones como son la posición en prono en Trendelenburg a $20^{\circ}$ de forma que se consigue abrir el ángulo del infundíbulo del polo inferior $10^{\circ}$ (35) y la posición de flanco que mejora el drenaje por gravedad de las litiasis a la pelvis renal favoreciendo su fragmentación y extracción, aunque hay que tener especial cuidado para minimizar la exposición a la radiación debido a la rotación lateral del arco en C (36).

En primer lugar se realiza una cistoscopia y se colocan 2 guías (Figura 7). Sobre una de ellas se procede a dilatación del ureter intramural con balón de dilatación de alta presión. Sobre una de las guías se coloca una vaina de acceso ureteral con un calibre de 12-14F. que facilita la introducción repetida del ureteroscopio flexible que será necesario para la extracción de los fragmentos después de su fragmentación (37) (Figura 8). El avance de la vaina de acceso ureteral se controla con el amplificador de imágenes. Cuando se comprueba su correcto posicionamiento se extrae el dilatador interno y la guía a través de la cual se ha colocado. Utilizamos la vaina de acceso ureteral Cook Flexorsheath (Cook Urological, Spencer, Indiana) debido a que estudios in vitro han demostrado que no se dobla después de la retirada del dilatador interno (38). Se introduce el ureteroscopio flexible. Después de la identificación del cálculo se introduce la fibra de láser de Holmium de 200 micras con el extremo del ureteroscopio recto para evitar dañar el canal de trabajo (39) (Figuras 9 y 10); se usa una energía de 0,5-1,2 J y una frecuencia de 5-15 Hz. Con esta fibra de láser sólo se pierde un 7-16\% de deflexión

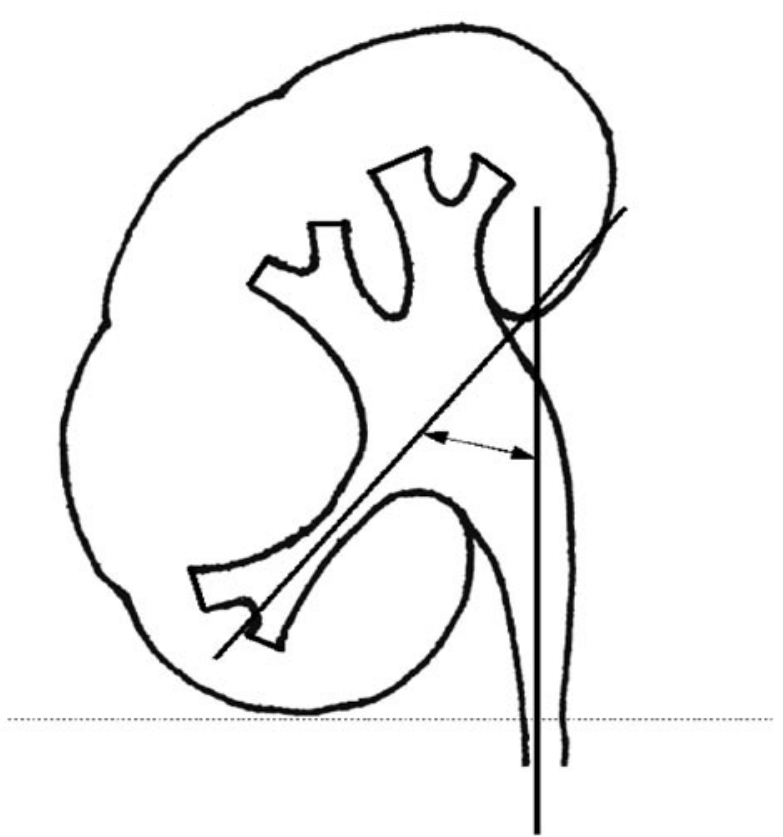

FIGURA 5. Ángulo infundibulo piélico de Bagley.

(-9ㅇ a $\left.-19^{\circ}\right)$ frente al $37 \%\left(-24^{\circ}\right.$ a $\left.-45^{\circ}\right)$ con la fibra de 365 micras $(39,40)$. También se puede desplazar la litiasis a la pelvis renal con una cesta de nitinol de calibre de 1,9 Fr mejorando la fragmentación y el rango de "stone free" y posiblemente la vida media del ureteroscopio flexible (41, 42). Preferimos una cesta de nitinol para esta maniobra debido a que es poco probable que se dañe la papila renal y se puede abrir entera (43). Cuando se utiliza una cesta de nitinol y si la entrada del líquido de irrigación o

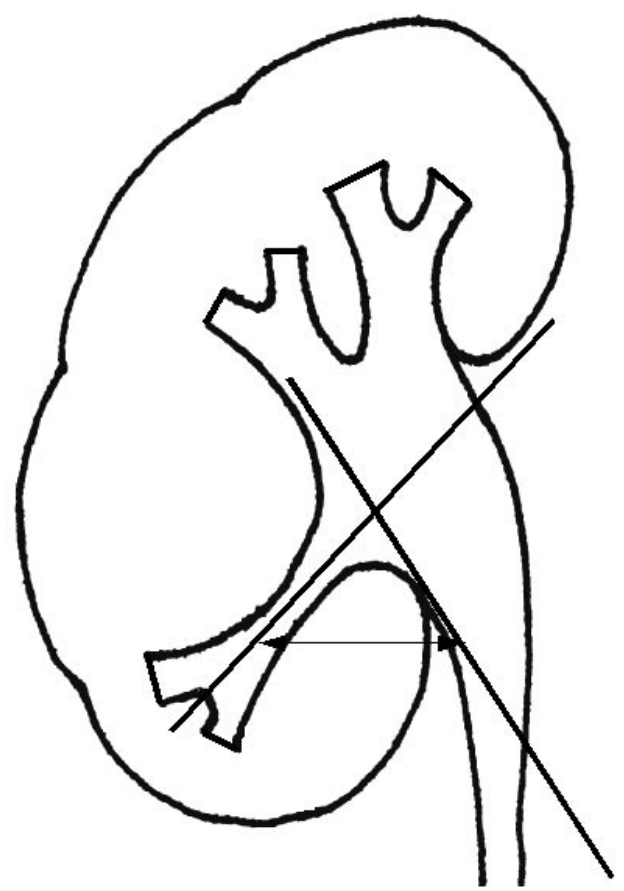

FIGURA 6. Ángulo infundibulo-piélico de Sampaio. 


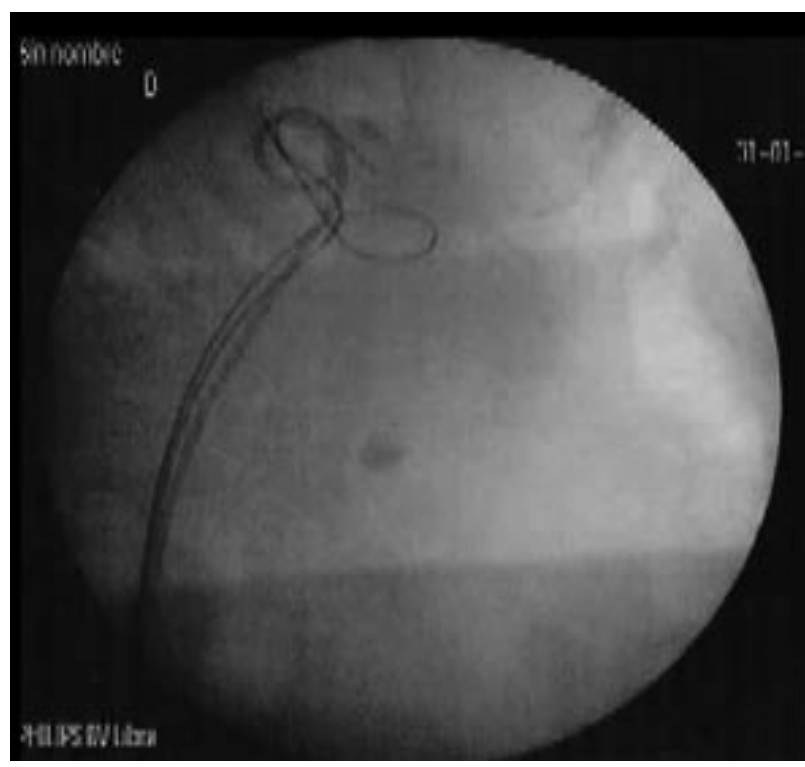

FIGURA 7. En el amplificador de imágenes se observan dos guías situadas en el sistema pielocalicial.

la deflexión que se alcanza no es suficiente se desmonta la misma $(44,45)$. Posteriormente al finalizar el procedimiento se deja un catéter doble $\mathrm{J}$ que se retira a la semana.

La revisión de la literatura aconseja la realización de URS flexible en las litiasis del polo inferior menores de 1 $\mathrm{cm}$, en los fracasos de la LEC o como primera modalidad de tratamiento en cálculos con niveles de atenuación mayores a $1000 \mathrm{HU}$, sospecha de que sean de cistina, pacientes obesos o con alteraciones de la coagulación (46).

\section{- Nefrolitotomía Percutánea (NLPC)}

Como ya se ha comentado anteriormente, basados en los resultados del meta-análisis de Lingenman y cols., mostrando un rango de "stone free" mayor para la

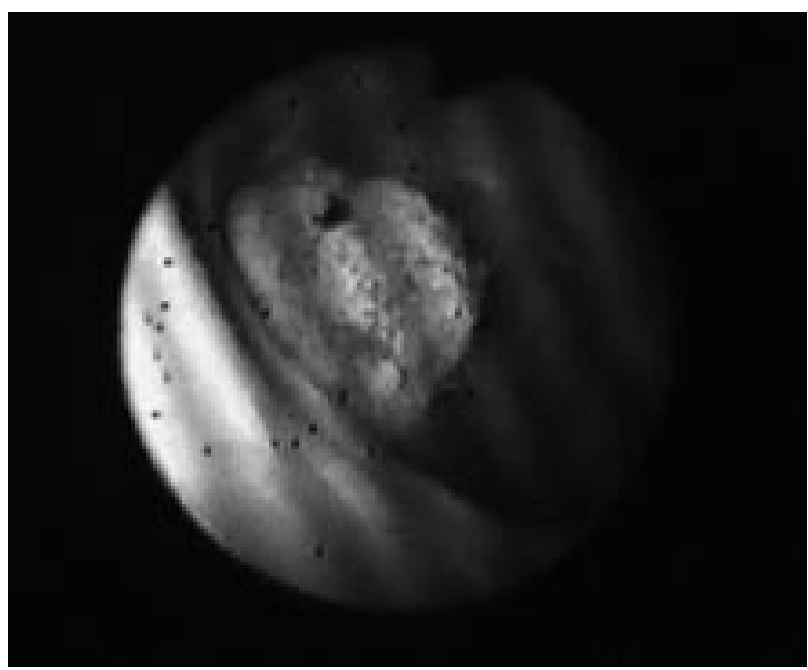

FIGURA 9. Se visualiza una litiasis en grupo calicial inferior.

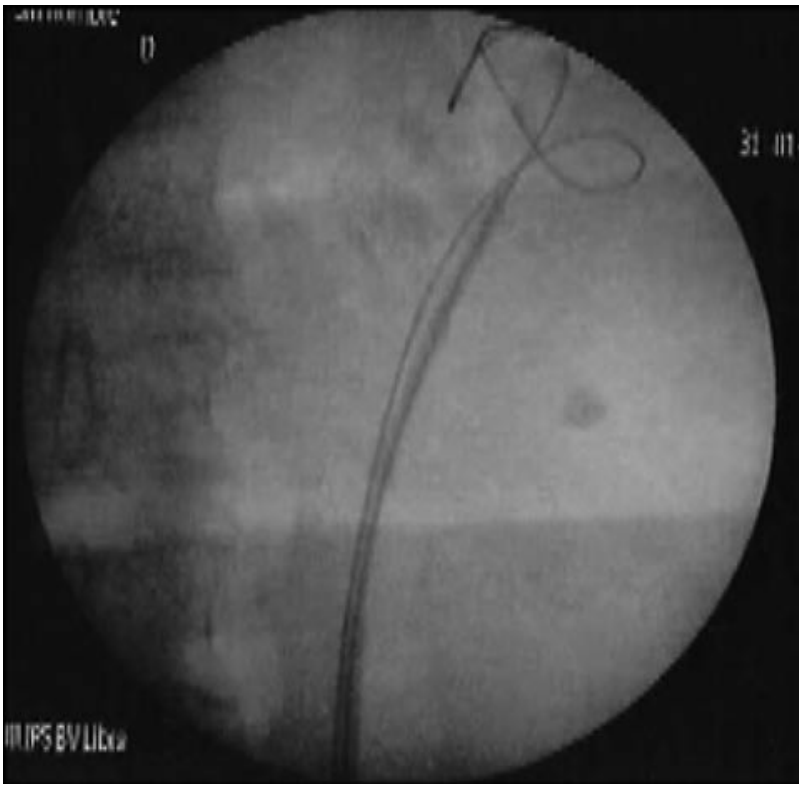

FIGURA 8. Sobre una de las guías se coloca la vaina de acceso ureteral.

NLPC que para la LEC para el tratamiento de las litiasis del polo inferior de diferente tamaño y validado por estudios prospectivos randomizados, se sugiere, que los pacientes con litiasis mayores de $1 \mathrm{~cm}$ deben ser tratados con NLPC $(4,5,46)$.

\section{ALGORITMOS DE DECISIONES TERAPEÚTICAS}

- "Lower pole ratio": es el cociente entre la longitud y la anchura infundibular. Los pacientes que tienen una "lower pole ratio" menor de 3,5 presentan un rango de "stone free" mayor con la LEC (47).

- Indice LC: Leykamm y cols. establecen una fórmula matemática utilizando la longitud infudibular y la altura pieloca-

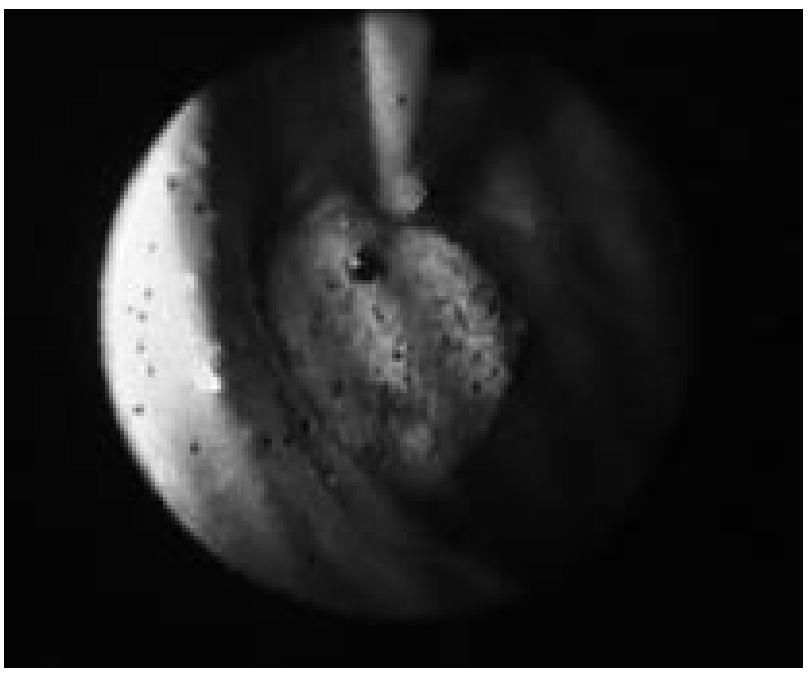

FIGURA 10. Se fragmenta la litiasis con una fibra de láser de Holmium-YAG de 200 micras. 


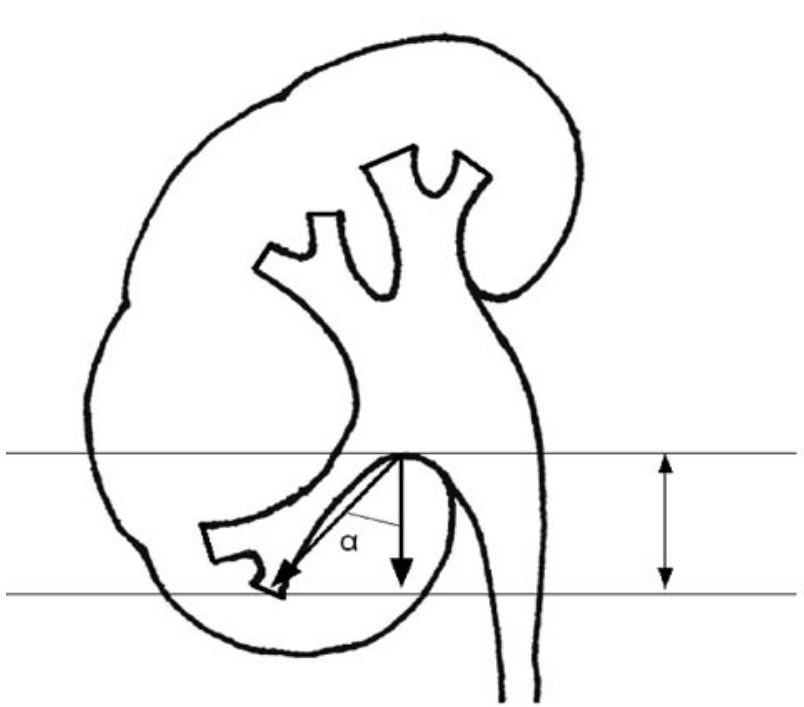

FIGURA 11. Algoritmo de Leykamm y cols.

licial. Definen el que viene definido como el cuadrado de la altura pielocalicial dividido por la longitud infundibular. Observan que si este índice es mayor del $25 \%$ existe una menor probabilidad de "stone free" en los pacientes tratados con LEC (48).

\section{- Índice de aclaramiento litiásico (SCI):}

Desai y cols. establecen la siguiente fórmula (49):

$\mathrm{SCl}=\left[\left(\mathrm{IVA} \times \mathrm{IW} \times\right.\right.$ tipo de litiasis $\left.\left.{ }^{*}\right) / \mathrm{IH}\right]-($ tamaño de la litiasis en $\mathrm{mm} 2$ / 10)

*Multiplicado por 1 para cálculos blandos y por 0,85 para cálculos duros.

$\mathrm{SCl}=$ índice de aclaramiento litiásico

IVA = ángulo infundibulo vertical

IW = anchura infundibular

$\mathrm{IH}=$ altura calicial

Según estos autores es posible predecir el éxito o no de la LEC en el tratamiento de la litiasis calicial inferior. Los pacientes con un $\mathrm{SCl}<-7$ deben conocer que la presencia de fragmentos es casi segura después del tratamiento con LEC, incluso aunque se trate de cálculos de pequeño tamaño, pudiendo aconsejar otras modalidades de tratamiento como la NLPC o la URS. Así litiasis de 2 $\mathrm{cm}$ de diámetro se podrían tratar con ESWL si el índice es positivo, pero se deberían considerar otras modalidades si es negativo.

\section{- Algoritmo de tratamiento en función del tamaño de la li- tiasis en polo inferior}

Wenn y cols. defienden la NLPC como primera línea de tratamiento en todas las litiasis del polo inferior mayores de $1 \mathrm{~cm}$. En las litiasis menores de $1 \mathrm{~cm}$ con características desfavorables (litiasis de cistina, litiasis con niveles de atenuación mayor a $1000 \mathrm{HU}$, distancia de la piel al cálculo mayor a $10 \mathrm{~cm}$ ) la primera línea de tratamiento debería ser la URS y en el resto la LEC (46).

\section{CONCLUSIONES}

El papel de la URS flexible en el tratamiento de la patología intrarrenal ha experimentado una importante evolución, impulsada por las mejoras en el diseño de los ureteroscopios flexibles, en su grado de deflexión y mejora de la calidad de imagen, en la gran diversificación de la intrumentación accesoria de pequeño calibre y en el uso del láser de Holmium (Ho: YAG) para la litotricia. Su desarrollo permite ofrecerla como modalidad terapeútica en los fracasos de la LEC en litiasis menores de $1 \mathrm{~cm}$ y como primera línea de tratamiento en las litiasis menores de $1 \mathrm{~cm}$ en casos de cálculos de cistina y en aquellos con niveles de atenuación mayores a $1000 \mathrm{HU}$; así como en pacientes obesos o con problemas de coagulación.

\section{BIBLIOGRAFIA Y LECTURAS RECOMENDADAS ( ${ }^{*}$ lectura de interés $y^{* *}$ lectura fundamental)}

1. HAVEL, D.; SAUSSINE, C.; FATH, C. y cols.: "Single stones of the lower pole of the kidney". Eur. Urol., 33: 396, 1998.

2. PACIK, D.; HANAK, T.; KUMSTAT, P. y cols.: "Effectiveness of SWL for lower-pole caliceal nephrolithiasis: Evaluation of 452 cases". J. Endourol., 11: 305, 1997.

3. CHEN, R.N.; STREEM, S.B.: "Extracorporeal shock wave lithotripsy for lower pole calculi: Long-term radiographic and clinical outcome". J. Urol., 156: 1572, 1996.

4. LINGEMAN, J.E.; SIEGEL, Y.I.; STEELE, B. y cols.: "Management of lower pole nephrolithiasis: A critical analysis". J. Urol., 151: 663, 1994.

*5. ALBALA, D.M.; ASSIMOS, D.G.; CLAYMAN, R.V. y cols.: "A prospective randomized trial of extracorporeal shock wave lithotripsy and percutaneous nephrostolithotomy for lower pole nephrolithiasis-initial results". J. Urol., 166: 2072, 2001.

6. KAYE, K.W.: "Renal anatomy for endourologic stone removal". J. Urol., 130: 647, 1983.

7. KAYE, K.W.; REINKE, D.B.: "Detailed caliceal anatomy for endourology". J. Urol., 132: 1085, 1984.

8. BAGLEY, D.H.; RITTENBERG, M.H.: "Intrarenal dimensions: Guidelines for flexible ureteropyeloscopes". Surg. Endosc., 1: 119, 1987.

9. SAMPAIO, F.J.B.; ARAGAO, A.H.M.: "Inferior pole collecting system anatomy: Its probable role in extracorporeal shock wave lithotripsy". J. Urol., 147: 322, 1992.

*10. NAKADA, S.Y.; HOFF, D.G.; ATTAI, S. y cols.: "Determination of stone composition by noncontrast spiral computed tomography in clinical setting". Urology, 55: $816,2000$.

**11. ZARSE, C.A.; McATEER, J.A.; TAN, M. y cols.: "Helical computed tomography accurately reports urinary stone composition using attenuation values: In vitro verification using high-resolution micro-computed tomogrphy calibrated to fourier transform infrared microspectroscopy". Urology, 63: 828, 2004.

12. JOSEPH, P.; MANDAL, A.K.; SINGH, S.K. y cols.: "Computerized tomography attenuation value of renal calculus: Can it predict successful fragmentation of the calculus by extracorporeal shock wave lithotripsy? A preliminary study". J. Urol., 167: 1968, 2002.

*13. GUPTA, N.P.; ANSARI, M.S.; KESARVANI, P. y cols.: "Role of computed tomography with no contrast 
medium enhancement in predicting the outcome of extracorporeal shock wave lithotripsy for urinary calculi". BJU Int. 95: 1285, 2005.

14. SAMPAIO, F.J.; D'ANUNCIACAO, A.L.; SILVA, E.C.: "Comparative follow-up of patients with acute and obtuse infundibulum-pelvic angle submitted to extracorporeal shockwave lithotripsy for lower caliceal stones: Preliminary report and proposed study design". J. Endourol., 11: 157, 1997.

15. ELBAHNASY, A.M.; CLAYMAN, R.V.; SHALHAV, A.L. y cols.: "Lower-pole caliceal stone clearance after shockwave lithotripsy, percutaneous nephrolithotomy, and flexible ureteroscopy: Impact of radiographic spatial anatomy". J. Endourol., 12: 113, 1998.

16. MAY, D.J.; CHANDHOKE, P.S.: "Efficacy and cost effectiveness of extracorporeal shock wave lithotripsy for solitary lower pole renal calculi”. J. Urol., 159: 24, 1998.

**17. PAREEK, G.; HEDICAN, S.P.; LEE, F.T. y cols.: "Shock wave lithotripsy success determine by skin to stone distance on computed tomography". Urology, 66: 941, 2005.

18. SCHULZ, E.; HENGST, E.; BRUNDIG, P. y cols.: "Disturbed urinary transport in the pelvi-calyceal system in calcium oxalate stone patients". Urol. Res., 15: 109, 1987.

19. MADBOULY, K.; SHEIR, K.Z.; ELSOBKY, E.: "Impact of lower pole renal anatomy on stone clearance after shock wave lithotripsy: Fact or friction?". J. Urol., 165: 1415, 2001.

20. TUCKEY, J.; DEVASIA, A.; MURTHY, L. y cols.: "Is there a simpler method for predicting lower pole stone clearance after shockwave lithotripsy than measuring infundibulopelvic angle?". J. Endourol., 14: 475, 2000.

21. ELBAHNASY, A.M.; SHALHAV, A.L.; HOENIG, D.M. y cols.: "Lower caliceal stone clearance after shock wave lithotripsy or ureteroscopy: The impact of lower pole radiographic anatomy". J. Urol., 159: 676, 1998.

22. SAMPAIO, F.J.B.: "Spatial anatomy of the lower calices: Importance in extracorporeal shock wave lithotripsy". Renal Anatomy Applied to Endourology and Interventional Radiology, Edited by F.J.B. SamDaio and R. Uflacker. New York: Thieme Medical-Publishers, pág. 16-22, 1993.

23. SAMPAIO, F.J.B.; ARAGAO, A.H.M.: "Limitations of extracorporeal shock wave lithotripsy for lower caliceal stones: Anatomic insight”. J. Endourol., 8: 241, 1994.

24. ANDERSSON, L.; SYLVEN, M.: "Small renal caliceal calculi as a cause of pain". J. Urol., 130: 752, 1983.

25. BRANDT, B.; OSTRI, P.; LANGE, P. y cols.: "Painful caliceal calculi. The treatment of small nonobstructing caliceal calculi in patients with symptoms". Scand. J. Urol. Nephrol., 27: 75, 1993.

26. MEE, S.L.; THUROFF, J.W.: "Small caliceal stones: Is extracorporeal shock wave lithotripsy justified?". J. Urol., 139: 908, 1988.

27. KEELEY, F.X. Jr.; TILLING, K.; ELVES, A. y cols.: "Preliminary results of a randomized controlled trial of prophylactic shock wave lithotripsy for small asymptomatic renal calyceal stones". BJU Int., 87: 1, 2001.

28. HUBNER, W.; PORPACZY, P.: "Treatment of caliceal calculi". Br. J. Urol., 66: 9, 1990.

29. GLOWACKI, L.S.; BEECROFT, M.L.; COOK, R.J. y cols.: "The natural history of asymptomatic urolithiasis". J. Urol., 147: 319, 1992.

*30. BURGHER, A.; BEMAN, M.; HOLTZMAN, J.L. y cols.: "Progression of nephrolithiasis: Long-term out- comes with observation of asymptomatic calculi”. J. Endourol., 18: 534, 2004.

*31. INCI, K.; SAHIN, A.; ISLAMOGLU, E. y cols.: "Prospective long-term followup of patients with asymptomatic lower pole caliceal stones". J. Urol., 177: 2189, 2007.

32. SAW, K.C.; LINGEMAN, J.E.: "Lesson 20d. Management of calyceal stones". AUA Update series, 20: 154, 1999.

**33. PEARLE, M.S.; LINGEMAN, J.E.; LEVEILLEE, R. y cols.: "Prospective randomized trial comparing shock wave lithotripsy and ureteroscopy for lower pole caliceal calculi $1 \mathrm{~cm}$ or less". J. Urol., 173: 2005, 2005.

34. GRASSO, M.; FICAZZOLA, M.: "Retrograde ureteropyeloscopy for lower pole caliceal calculi". J. Urol., 162: 1904, 1999.

35. BERCOWSKY, E.; SHALHAV, A.L.; ELBAHNASY, A.M. y cols.: "The effects of patient position on intrarenal anatomy". J. Endourol., 13: 257, 1999.

36. HERRELL, S.D.; BUCHANAN, M.G.: "Flank position ureterorenoscopy: New positional approach to aid in retrograde caliceal stone treatment". J. Endourol., 16: $15,2002$.

37. MONGA, M.; BHAYANI, S.; LANDMAN, J. y cols.: "Ureteral access for upper urinary tract disease: The access sheath". J. Endourol., 15: 831, 2001.

38. GAWLIK, A.; DURFEE, W.; MONGA, M.: "Physical characteristics of ureteral access sheaths". J. Endourol., 16: 26, 2002.

39. KUO, R.L.; ASLAN, P.; ZHONG, P. y cols.: "Impact of holmium laser settings and fiber diameter on stone fragmentation and endoscope deflection". J. Endourol., 12: 523, 1998.

40. KOURAMBAS, J.; BYRNE, R.R.; PREMINGER, G.M.: "Does a ureteral access sheath facilitate ureteroscopy?”. J. Urol., 165: 789, 2001.

41. AUGE, B.K.; DAHM, P.; WU, N.Z. y cols.: "Ureteroscopic management of lower-pole renal calculi: Technique of calculus displacement". J. Endourol., 15: 835, 2001.

42. PIETROW, P.K.; AUGE, B.K.; DELVECCHIO, F.C. y cols.: "Techniques to maximize flexible ureteroscope longevity". Urology, 60: 784, 2002.

43. HONEY, R.J.D.: "Assessment of a new tipless nitinol stone basket and comparison with an existing flatwire basket". J. Endourol., 12: 529, 1998.

44. LANDMAN, J.; MONGA, M.; EL-GABRY, E.A. y cols.: "Bare naked baskets: Ureteroscope deflection and flow characteristics with intact and disassembled ureteroscopic nitinol stone baskets". J. Urol., 167: 2377, 2002.

45. BHAYANI , S.B.; MONGA, M.; LANDMAN, J. y cols.: "Bare naked baskets: Optimizing ureteroscopic stone extraction". Urology, 60: 147, 2002.

46. WEN, C.C.; NAKADA, S.Y.: "Treatment selection and outcomes: Renal calculi". Urol. Clin. North Am., 34: 409, 2007.

*47. FONG, Y.K.; PEH, S.O.; HO, S.H. y cols.: "Lower pole ratio: A new and accurate predictor of lower pole stone clearance after shockwave lithotripsy?". Int. J. Urol., 11: 700, 2004.

*48. LEYKAMM, L.; TISELIUS, H.G.: "Observations on intrarenal geometry of the lower-caliceal system in relation to clearance of stone fragments after extracorporeal shockwave lithotripsy". J. Endourol., 21: 386, 2007.

*49. DESAI , M.R.; RAGHUNA,T.H.; MANOHAR, T. y cols.: "Lower-caliceal stone clearance index to predict clearance of stone after SWL". J. Endourol., 20: 248, 2006. 